\title{
Un inventario biológico para México
}

\author{
RODOLFO DIRZO ${ }^{1}$ Y PETER H. RAVEN ${ }^{2}$ \\ ${ }^{\prime}$ Centro de Ecología, UNAM. Apdo. Postal 70-275, 04510 México D.F. \\ ${ }^{2}$ Missouri Botanical Garden, P.O. Box 299, St. Louis MO 63166-0299
}

USA

\begin{abstract}
Resumen. Los recursos biológicos de México constituyen el mayor recurso renovable sobre el cual descansa, en última instancia, la productividad natural del país. El conocimiento acerca de estos recursos es limitado, disperso y a menudo difícil de obtener. En esta era de creciente interés de la sociedad en general por los llamados problemas ambientales, así como de evidente deterioro de los ecosistemas naturales del país, el déficit de información básica sobre la riqueza, distibución y composición de la biota de México se hace aún más evidente. El afrontar este problema de manera directa, a través de la iniciación de un proyecto del inventario biológico del país, sería una labor de importancia científica y económica considerables para el país. El propósito de este ensayo es describir la importancia de un proyecto de prioridad nacional, el Inventario Biológico de México. La Sociedad Botánica de México, una de las sociedades científicas de mayor tradición en México, puede jugar un papel preponderante en la reflexión, guía y desarrollo de lo que falta por hacer en un proyecto de importancia nacional como el que aquí discutimos.
\end{abstract}

Abstract. The biological resources of Mexico constitute the most important renewable resource upon which the natural productivity of the country rests. Knowledge about these resources is limited, and often difficult to obtain. In these times of increasing public interest for the so-called environmental problems, as well as of increasing degradation of the country's natural ecosystems, the deficit of basic information about the richness, composition and distribution of Mexico's biota is even more evident. To face this problem directly, through the initiation of a project of biological inventory of Mexico, would be a task of considerable scientific and economic importance for the country. The aim of this essay is to describe the importance of a project of national priority, the biological inventory of Mexico. The Botanical Society of Mexico may play a crucial role in the promotion, reflection, guidance and development of the pending aspects of a project of such national priority like the one we discuss here.

\section{INTRODUCCIÓN}

Los recursos biológicos de México -plantas, animales, hongos y microorganismos- constituyen el mayor recurso renovable sobre el cual descansa, en última instancia, el bienestar del país. El conocimiento acerca de estos recursos es limitado, disperso y a menudo difícil de obtener, de tal suerte que las formas más apropiadas de conservarlos y utilizarlos son frecuentemente entorpecidas. En esta era de creciente interés popular y, aparentemente, gubernamental, por los llamados problemas ambientales (p. ej. el establecimiento del día mundial del ambiente por parte de la ONU, y celebrado bajo el patrocinio del gobierno de México en junio, 1990; la celebración de la Conferencia Mundial del Ambiente, en Río, en 1992, etc.), el déficit de información básica sobre la riqueza y composición de la biota de México se hace aún más evidente. El afrontar este problema de manera directa, a través de la iniciación de un proyecto del inventario biológico del país, sería una acción de importancia científica y económica considerable. El propósito de este ensayo es describir la importancia de un proyecto de prioridad nacional, el Inventario Biológico de México. Aquí delineamos las bases conceptuales subyacentes a dicho proyecto.

Las plantas, animales, hongos y microorganismos, como entes individuales, despliegan sus atributos morfológicos y fisiológicos gracias a su constitución genética. Al interacuar entre ellos y con el ambiente físico, constituyen las comunidades naturales del país y determinan su funcionamiento. Las modificaciones de estas comunidades naturales tales como su reemplazamiento por campos agrícolas o forestales, pueden ser exitosas, en parte, debido a la aplicación del conocimiento derivado de los sistemas naturales que son reemplazados. Además, aun sin ser reemplazados por algún tipo de agroecosistema, los sistemas naturales son con frecuencia objeto de cosecha o extracción de, por ejemplo, leña, plantas medicinales, animales (incluyendo insectos) para consumo, y otros varios productos naturales. La sostenibilidad de tales actividades depende hasta cierto punto de la aplicación del conocimiento disponible (científico y aún empírico) acerca del funcionamiento de las comunidades bajo explotación, lo cual a su vez depende del conocimiento del funcionamiento de los organismos individuales y sus poblaciones.

Además del papel que juegan en el funcionamiento del ecosistema a través de procesos tales como el reciclaje de nutrimentos (ver p. ej. Herrera et al., 1981) y la determinación de presupuestos de gases atmosféricos (p. ej. Masera et al., 1992); en la protección de cuencas, suelos y agua de los que depende el país (véase p. ej. Maass et al., 1988), las comunidades naturales consisten de una constelación de 
organismos que, como fuente renovable de productos naturales, son útiles individualmente. Ejemplos de esto lo constituyen la extracción de madera de ciertas especies comerciales (caoba, cedro, hojas de palma), el uso de numerosas plantas nativas como fuente de medicina, y el consumo de insectos, peces y mamíferos en varias comunidades del país. A medida que aumenta el conocimiento biológico y etnobiológico de los organismos, muchas más propiedades se podrían descubrir, las cuales se podrían aplicar para beneficio humano en el país. Por ejemplo, tan solo a partir de la Segunda Guerra Mundial, se han patentado unas 3, 000 clases de antibióticos (Raven et al., 1981) y, sin duda, muchas más se podrían derivar a partir de una biota tan rica como la de México. La medida en que ésto se pueda lograr depende, a su vez, del grado en que los organismos sean conocidos y estudiados. En una era de tecnología genética tan avanzada como ésta, es posible transferir genes de un organismo a otro y por lo tanto transferir propiedades útiles a organismos de interés económico. Esto implica que, dada la amenaza de extinción biológica contemporánea, por cada población o especie de organismo que se pierde (se extingue), perdemos no sólo esa entidad biológica particular con las propiedades que posee o integra en su propia morfología y funcionamiento, sino que además perdemos decenas de miles de genes, algunos de ellos potencialmente útiles.

\section{LA NECESIDAD DE UN INVENTARIO BIOLÓGICO NACIONAL}

Los argumentos de la importancia económica de catalogar, usar y conservar la biota de México deberían ser evidentes a partir de los comentarios anteriores. Sin embargo, otra línea de argumentación que queremos reiterar es que una plétora de organismos se encuentran en inminente riesgo de perderse como consecuencia de diversas actividades humanas contemporáneas. Una sólida base de datos referente a las plantas, animales, hongos y microorganismos del país es esencial para preservarlos. Existen varias estrategias para la preservación biológica, y la selección de la estrategia más pertinente, así como la selección más eficiente de organismos particulares, dependen de nuestro entendimiento de lo que son los organismos y dónde se encuentran. La protección total de áreas selectas es una de las formas más obvias de preservar constelaciones de organismos, pero la selección de tales áreas depende de la aplicación eficiente de la información acerca de la distribución y abundancia de los organismos. Además, la preservación de algunos organismos es más apropiada específicamente o, adicionalmente, fuera de los sitios donde ocurren naturalmente, ya sea porque estos sitios están siendo destruídos, o porque las condiciones de los mismos están siendo cambiadas a tal punto que los organismos, individualmente, no pueden sobrevivir, o porque las poblaciones de los mismos son tan bajas que en condiciones naturales se encuentran amenazados por la simple ocurrencia de cambios aleatorios (o aún impredecibles) del ambiente.
Hasta hace poco, aparte de las actividades que en este sentido ha venido llevando a cabo la Comisión Nacional para el Conocimiento y Uso de la Biodiversidad (CONABIO), no se había intentado ningún esfuerzo coordinado por sondear y catalogar el total de la biota de México. Algunos esfuerzos anteriores notables se han llevado a cabo a nivel regionoal y en particular con plantas. El ejemplo pionero por excelencia es la flora del Estado de Veracruz (GómezPompa y Nevling, 1970), y recientemente, la Flora Mesoamericana. Para muchos grupos de organismos, incluyendo, por ejemplo, invertebrados (notablemente insectos), hongos y microorganismos, no ha habido trabajo de inventarización y descripción para muchas regiones extensas del país. Incluso algunos grupos de importancia económica obvia han pasado desapercibidos en los intentos previos de inventarización. Un ejemplo de ésto es el de las plantas que constituyen los ancestros silvestres de plantas cultivadas. Notable en ésto es el revolucionario descubrimiento, apenas hacia el final de los 70's, del maíz perenne por Rafael Guzmán (Guzmán, 1977; Iltis et al., 1979). Esta especie, que ocurre en un área limitada de una región que hasta hace poco se encontraba en riesgo de alteración y posible destrucción, es de enorme importancia económica (por su potencial como fuente de transferencia de genes de resistencia a patógenos y de su carácter perenne). Este caso ofrece una clara indicación de las muchas otras especies de plantas (tales como leguminosas, pastos, o palmas) y animales (por ejemplo insectos) que aguardan a ser descubiertos y que podrían ser de gran importancia.

Además, incluso las biotas de áreas que han sido formalmente protegidas y relativamente bien estudiadas biológicamente, se encuentran sólo parcialmente inventariadas, ya que la mayoría de los inventarios se han concentrado en los organismos más conspicuos o «más atractivos» (p. ej. aves, árboles). Por ejemplo, en la Estación Biológica de Los Tuxtlas, Veracruz, algunos intentos recientes por inventariar los patógenos foliares que son transmitidos por insectos fitófagos, resultaron en la recolección de una gran cantidad de patógenos que no son identificables por especialistas y que sin duda resultarán en especies nuevas (GarcíaGuzmán, 1990). Una situación comparable ha sido documentada en un estudio de los insectos depredadores de semillas de la flora arbórea de la misma estación biológica (Andrade, 1989), en el cual numerosas especies han resultado nuevas para la ciencia. Lo mismo podría decirse de muchos otros grupos y de otras áreas. La realización de un inventario, lo más completo posible, podría jugar un papel crucial en el desarrollo subsecuente de la investigación científica en las ciencias biológicas, y tendría un fuerte impacto en el desarrollo ecológicamente compatible de muchas áreas.

La importancia de llevar a cabo un inventario de magnitud nacional no es exagerada en vista de la rápida y a menudo permanente y semipermanente destrucción de las comunidades naturales de México-sus recursos naturales. En las partes tropicales húmedas del país, donde ocurre la 
deforestación más intensa, del orden del $2 \%$ por año (Masera et al., 1992), se hace evidente la extrema urgencia de llevar a cabo un proyecto de inventario. antes de que sea demasiado tarde. Asimismo, de acuerdo a las estimaciones de Rzedowski y Calderón de Rzedowski (1987), el bosque tropical seco de la extensa región del Bajío, ha sido casi totalmente destruido, y el trabajo de inventarización ha quedado muy rezagado. Lo mismo podría decirse de muchos de los bosques tropicales secos localizados a lo largo de la costa del Pacífico de México. En contraposición a esto, menos del 5\% del territorio nacional ha sido incorporado en el sistema de áreas protegidas que efectivamente funcionen como tales (FloresVillela y Geréz, 1988; A. Gómez-Pompa y R. Dirzo, datos no publicados). Esto indica, una vez más, la imperiosa necesidad de un inventario biológico nacional que opere como una herramienta para el manejo tanto de áreas naturales (que permitan las mejores y más eficientes decisiones posibles respecto a las áreas que deberán ser protegidas), así como de las especies que son más importantes y en peligro más inmediato de erradicación local o extinción en general (véase Vovides, 1981).

Por otra parte, el trabajo de inventario biológico constituye el fundamento científico para la definición de las áreas con potencial de incorporarse al sistema de áreas protegidas. Por ejemplo, algunos estudios recientes sobre las selvas secas del país, revelan que aunque la riqueza de especies de plantas leñosas de una localidad dada no difiere sustancialmente de la de otra localidad contigua, la proporción de especies compartidas es muy pequeña (R. I. Trejo, datos no publicados). De esta manera, por ejemplo, la selección de sólo una de las dos localidades en cuestión para hacerla un área protegida, podrá tener potencial para proteger el tipo de ecosistema, pero no necesariamente de las especies.

Además, desde luego, la importancia de una apreciación y conocimiento crecientes de los recursos biológicos de México para la educación, tanto formal como informal, es crucial.

\section{LA MAGNITUD DE LOS RECURSOS BIOLÓGICOS DE MÉXICO}

Es importante enfatizar que México es asiento de una extraordinariamente rica constelación de tipos de ecosistema, así como de especies de plantas, animales, hongos y microorganismos. Con base en los datos disponibles hasta ahora, para el caso de las plantas vasculares, que suman un mínimo de 20-22, 000 especies de un total mundial de aproximadamente 250,000 (Rzedowski, 1991), y para el caso de vertebrados terrestres, que suman 2,401 especies registradas de un total mundial de aproximadamente 22,000 (Flores-Villela y Geréz, 1988), resulta obvio que México posee una riqueza biológica poco comparable con la de otros territorios de extensión equivalente. Esto es al punto tal que el territorio de México ha sido considerado como uno de los 10 sitios de megadiversidad a nivel planetario (Mittermeier y Goettsch de Mittermeier,
1992). Ya que estos valores sugieren que entre un 8 y un $10 \%$ de las plantas y vertebrados terrestres del planeta ocurren dentro del territorio de México, podría esperarse que pudiesen existir, dentro de los límites del país, un mínimo de 500,000 especies de todos los grupos. Estos datos, aun cuando son muy preliminares, son apoyados por los datos obtenidos a partir de inventarios biológicos detallados en zonas restringidas (váse por ejemplo, Ibarra y Sinaca, 1987). En cualquier caso, y aun con las estimaciones mínimas parecería claro que no más del $50 \%$ de los organismos que ocurren en México han sido identificados y caracterizados científicamente. Para muchos de otros organismos no tenemos información de ningún tipo, y sólo un mínimo han sido explorados en cuanto a su potencial de utilidad directa para el hombre.

Por otra parte, se estima que existe un mínimo de 3,624 especies de plantas vasculares que son endémicas de México (Rzedowski, 1991). Estos valores sugieren que aproximadamente un $1.5 \%$ de las plantas del planeta.(y quizá $3 \%$ de los vertebrados terrestres del mundo) se encuentran sólo en México. Esto sugire a su vez que, conservadoramente, probablemente un mínimo de 80,000 especies de organismos podrían ocurrir sólo dentro de los límites del territorio nacional. Es decir, se trata de una enorme proporción de la diversidad biológica del planeta, y de un recurso considerablemente importante para México en el futuro.

\section{EL INVENTARIO BIOLÓGICO DE MEXICO}

Para entender y valorar cabalmente esta riqueza biológica, sería de gran utilidad consolidar un Inventario Biológico Nacional de México, con la participación total de universidades, museos, las dependencias pertinentes del gobierno, así como organizaciones no gubernamentales que tengan capacidad en estas áreas. La reciente creación de la CONABIO, ya ha dado pasos de gran importancia en este sentido y opera, en la realidad, como el motor coordinador del proyecto. El inventario biológico nacional debería tener, como componente central, la construcción de un banco de datos computarizado sobre la ocurrencia y distribución de organismos en el país, incorporando además, los avances de los proyectos de este tipo que se encuentran ya en marcha. En este sentido, la comunidad botánica ha hecho avances notables entre los que destacan: el proyecto de la Flora de Veracruz (Gómez-Pompa y Nevling, 1970) que incluye una base de datos computarizada avanzada, y el proyecto de la Flora Mesoamericana, un proyecto conjunto de la UNAM, el Museo de Historia Natural de Londres y el Jardín Botánico de Missouri (Davidse et al., 1981), que incluye todas las plantas a partir del Itsmo de Tehuantepec hacia el sur, incorporando la información en un banco computarizado. La revitalización del comité sobre la Flora de México es otro esfuerzo que, bajo el auspicio de la Sociedad Botánica de México, podría continuar y convertirse en un componente crucial del inventario. Otros numerosos esfuerzos preliminares se han iniciado en diferentes partes del país, en gran 
medida con el apoyo de la CONABIO, y son piezas clave que no deben abandonarse y llevarse hasta el punto de consolidación de la gran base computarizada del inventario.Todas estas actividades, junto con el creciente interés gubernamental y del público en general sobre los problemas ambientales, sugieren que la consolidación de un inventario biológico nacional es un proyecto lógico y absolutamente necesario.

Otras condiciones favorables para el establecimiento del proyecto incluyen la considerable tradición científica en los campos pertinentes al mismo, incluyendo las actividades y escuelas generadas por biólogos tales como Faustino Miranda, Efraím Hernández-X, Jerzy Rzedowski, Eizi Matuda, Helia Bravo y Arturo Gómez-Pompa (ver análisis históricos en Rzedowski, 1981; Sarukhán, 1981). Una tradición zoológica equivalente se debe principalmente a personas como Gonzalo Halffter, Alfredo Barrera y Bernardo Villa. Además, el desarrollo de la ciencia en México, en estas áreas, ha estado abierto a la participación de científicos de otros países que han hecho contribuciones importantes (por ejemplo A. J. Sharp, H. S. Gentry, R. Mc. Vaugh y H. Smith) y esta colaboración no debería abandonarse. Por otra parte, actualmente existen en México un número de instituciones sólidas con una participación activa en las áreas pertinentes al desarrollo del proyecto, incluyéndose el Centro de Ecología (UNAM), el Instituto de Biología (UNAM) y la Facultad de Ciencias (UNAM), el Instituto de Ecología, el Colegio de Posgraduados y la Universidad de Chapingo, el CIMMyT, así como varias universidades estatales y un vigoroso grupo de estudiantes y científicos jóvenes con capacidades pertinentes al desarrollo del proyecto.

Un aspecto de la situación general que debe considerarse seriamente es el tremendo conocimiento trádicional y de uso de los recursos biológicos por grupos o culturas nativas de México (véase Hernández-X., 1985). Este conocimiento, que ha sido desarrollado a gran escala pero que se encuentra en un proceso de rápida erosión en algunas partes del país debería ser una pieza central en el desarrollo de un inventario nacional y debería ser incorporado del todo en la concepción y alcances del inventario, con ventajas adicionales considerables para el país.

\section{NATURALEZA Y ALCANCES DEL INVENTARIO BIOLÓGICO}

El Inventario Biológico de México propuesto aquí, como se ha discutido, jugará un papel central en el desarrollo de las ciencias biológicas y en la utilización sostenible, económicamente sólida de los recursos biológicos del país. El proyecto debe incluir un número de componentes entre los que destacan como básicos la recolección y preservación de especímenes, la provisión de medios adecuados para su conservación y almacenamiento en forma segura y permanente, el desrrollo de un banco de datos computarizado que incorpore la información de las recolecciones recientes, así como de aquellas que se han hecho anteriormente y que se encuentran almacenadas en México o en el extranjero, y el desarrollo de sistemas para el manejo de información, incluyéndose una red de información computarizada. Junto con la CONABIO, el desarrollo de un Museo Nacional de Historia Natural como el que se había propuesto hace unos años por parte de la UNAM, pudieran proveer el sitio adecuado para almacenamiento y establecimiento de dichas facilidades.

Una vez que se complete, la base de datos computarizada debería ser capaz de generar mapas de la distribución de todos los organismos para los cuales se tenga información. Tal información a su vez, debería utilizarse como base para llevar a cabo la exploración biológica subsecuente de las áreas menos conocidas del país, así como de aquellos ecosistemas que están siendo perturbados más intensamente o que estén en condiciones más críticas de amenaza de destrucción completa o semicompleta en el futuro cercano.

El banco de datos resultante de estos esfuerzos deberá incorporar información acerca de los usos económicos de los organismos y debería utilizarse como un método conveniente para el muestreo subsecuente de otros organismos con potencial de utilidad económica. El rico almacén de información sobre la utilización de las plantas de México por parte de diversas etnias y grupos rurales debe ser considerado y desarrollado plenamente en el banco de datos. Además, el banco de datos debería ser usado como una base para los planes de conservación. Algunas de las estrategias de conservación que en forma lógica resultarían de la presentación eficiente de la información de este tipo, serían la selección y preservación de áreas naturales, enfocadas específicamente a la preservación y valoración de la diversidad natural. El tipo de información integrada que sería posible generar con la base de datos del inventario biológico sería una de las mejores herramientas de selección de tales áreas.

Hasta hace poco, las áreas protegidas en México, como en otros países, se han venido seleccionando en gran medida debido a sus atributos físicos particulares tales como la presencia de lagos, cascadas, montañas y otros aspectos estéticos y no directamente debido a consideraciones ecológicas o de su diversidad biológica. Un esfuerzo nacional debería dirigirse para adquirir muestras representativas de los ecosistemas pobremente representados. Tales esfuerzos podrían hacerse más rápido a través de la creación del banco de datos generado por el inventario nacional. No es ocioso enfatizar que tales esfuerzos deben ser racionalizados, ya que muchas de las áreas biológicamente interesantes en términos de su diversidad y por ser únicas, pueden ser indistinguibles desde un punto de vista estético y bien podrían ser pasadas por desapercibido, mientras que hábitats completos, biológicamente interesantes, podrían ser erradicados a lo largo de su extensión total en el país.

A fin de llevar a cabo estas operaciones en forma eficiente, será necesario poner atención especial al estimular ecólogos y biólogos mexicanos interesados en la sistemática y proveer un número de puestos para ellos en universidades, museos y otras instituciones de investigación. El apoyo a los estudiantes de posgrado sería, igualmente, un aspecto importante en el desarrollo adecuado del programa. En térmi- 
nos del conocimiento de los diferentes grupos de organismos y de la capacidad nacional para lidiar con ellos, sería pertinente llevar a cabo un estudio de evaluación de la situación actual para planear la adición subsecuente de científicos que deberían ser reclutados en las áreas donde no hay especialistas, o si los hay, son muy pocos. Vale la pena pensar en la colaboración con instituciones e individuos del extranjero, para las áreas que se considere apropiado, pero la tarea fundamental del inventario biológico nacional debería hacerse con base en fortalecer la comunidad científica mexicana, de tal manera que sean los propios mexicanos los encargados de lidiar en forma más efectiva y eficiente con el manejo de los recursos biológicos del país. El entrenamiento de estudiantes especializados debería preverse, en algunos casos en el extranjero, con el fín de entrenar a científicos en formación para lidiar con aquellos grupos de organismos que son particularmente poco conocidos (por ejemplo hongos y microorganismos) y para los cuales no existe experiencia en el país.

Además del entrenamiento de científicos y la provisión de oportunidades adecuadas para emplearlos, el entrenamiento de individuos capacitados en la selcción y preparación de especímenes, a los que podríamos llamar paraprofesionales, debería ser estimulada a lo largo del país. El conjuntar un número adecuado de científicos especializados en la investigación pertinente al inventario biológico de México, bien podría ser una imposibilidad práctica y, al mismo tiempo, sería deseable entrenar a un número considerable de personas no necesariamente profesionales a que participen en el proyecto, de tal forma que una mayor representación de la comunidad mexicana pudiese participar.

Un banco nacional de propágulos (semillas, huevecillos de insectos, tejidos en cultivo, etc.) también podría ser incorporadao al proyecto de tal manera que los organismos particularmente amenazados o bien de interés económico especial, pudieran ser conservados en condiciones apropiadas. Duplicados de estos elementos podrían ser distribuidos en uno o más centros de bancos genéticos internacionales y nacionales por razones de seguridad de conservación de las fuentes genéticas involucradas. La existencia y funcionamiento del Centro Internacional para el Mejoramiento del Maíz y Trigo en Texcoco, es una experiencia valiosa que bien podría usarse como base para este fin.

La base de datos computarizada pertinente a los organismos de México, podría estar en capacidad de revelar el estado de conservación de cada organismo ya sea en la naturaleza, en cultivo, o en bancos genéticos y podría usarse como una base para acciones de conservación, en la cual se pudiera asignar prioridades a aquellos organismos particularmente útiles desde el punto de vista científico o económico. De nuevo, la existencia de instituciones tales como el CIMMyT es una experiencia interesante de partida. Asimismo, el desarrollo de facilidades similares para el caso de microorganismos, un aspecto pobremente desarrollado en México hasta ahora, debería considerarse como un mecanismo complementario a la selección y preservación de áreas naturales generadas a partir del banco de datos del inventario. Algunos inventarios biológicos similares están siendo desarrollados e implementados en Canadá (Danko, 1986), Australia (Bridgewater, 1986) Costa Rica (R. Gámez y D. H. Janzen, datos no publicados), China y Taiwan (P.H. Raven, datos no publicados), los EE UU (Kosztarab, 1984) y en algunas otras partes del mundo. La información y experiencia generadas debería compartirse con aquellos involucrados en el desarrollo de esfuerzos paralelos ya que mucho de lo que se ha aprendido podría aplicarse directa y efectivamente en México.

En resumen, la productividad natural de México -la base de la existencia humana- depende de las diversas comunidades biológicas que ocurren a lo largo y ancho del país, y de los organismos que junto con su ambiente conforman estas comunidades. Los recursos que los mexicanos tendrán para el bienestar de la gente en 50 ó 100 años o en el futuro indefinido, dependerá directamente de las acciones que se tomen ahora, y que estén dirigidas a establecer una base segura de conocimiento, uso y conservación de los organismos que ocurren en el país. La implementación urgente de un inventario biológico nacional, el cual proveería las bases para la racionalización de estas actividades, mejorando la calidad de recursos bibliotecarios y de almacenamiento de los especímenes en el país, así como la promoción del entrenamiento de otros científicos en las áreas pertinentes al inventario, debería llevarse a cabo como una política dirigida al interés nacional actual y con efectos de larga duración.

Dado que la parte correspondiente al acopio de la información disponible, promoción de la generación de nueva información, y la generación de bases de datos computarizadas como se ha esbozado en este ensayo, ha avanzado considerablemente a partir de la creación de la CONABIO, se requiere ahora de un esfuerzo sustancial acoplado, en lo referente a: las colecciones científicas (y sus acervos de literatura correspondientes), entrenamiento de personal en las áreas pertinentes, la promoción de la valoración cabal, por parte de la sociedad, de la importancia de los recursos biológicos del país, y la aplicación de los conocimientos generados. La Sociedad Botánica de México, una de las sociedades científicas de mayor tradición en México, puede jugar un papel preponderante en la reflexión, guía y desarrollo de lo que falta por hacer en un proyecto tan importante como el que aquí discutimos.

\section{AGRADECIMIENTOS}

José Sarukhán leyó una versión previa de este escrito y ofreció sugerencias valiosas. Guillermina Gómez nos ayudó en varios aspectos de la preparación del mismo.

\section{LITERATURA CITADA}

Andrade O. 1989. Cultivo de insectos depredadores de propágulos de árboles tropicales en Los Tuxtlas, Veracruz. Tesis de Licenciatura, Facultad de Ciencias, UNAM, México.

Bridgewater PB. 1986. The Australian biological resources study. En: 
Kim K.C, Knutson L, edrs. Foundations for a national biological survey. Assoc. Syst. Coll., Lawrence, KS, 167-176.

Danko, HV.1986. Biological survey of Canada (terrestrial arthropods). En: Kim KC, Knutson L, edrs. Foundations for a national biological survey. Assoc. Syst. Coll., Lawrence, KS, 203-208.

Davidse G, Sousa M, Humphries CJ, Chater AO. 1981. Flora Mesoamericana. Taxon 30: 710-713.

Flores-Villela O, Geréz P. 1988. Conservación en México. INIREBConservation International, Mexico.

García-Guzmán G. 1990. Estudios sobre ecología de patógenos en el follaje de la selva de Los Tuxtlas, Ver. Tesis de Maestría, Facultad de Ciencias, UNAM, México.

Gómez-Pompa A, Nevling LI. 1970. La flora de Veracruz. An. Inst. Biol. Univ. Nac. Méx. 4: 1-2.

Guzmán R. 1977. Redescubrimiento de Zea perennis (Graminae). Phytologia 38: 177.

Hernández-X E. 1985. Exploración etnobotánica y su metodología. Xolocotzia 1: 163-197.

Herrera R, Jordan CF, Medina E, Klinge H. 1981. How human activities disturb the nutrient cycles of a tropical rainforest in Amazonia. Ambio 10: 109-114.

Ibarra-Manríquez G, Sinaca S. 1987. Listados florísticos de México. VII. Estación de Biología Tropical Los Tuxtlas. Instituto de Biología, UNAM. México.

Iltis HH, Doebley JF, Guzmán R, Pazy B. 1979. Zea diloperennis (Graminae): A new teosinte from Mexico. Science 203: 186-188.

Kosztarab M. 1984. A biological survey of the United States. Science: 233: 443 .
Maass JM, Jordan CF, Sarukhán J. 1988. Soil erosion and nutrient losses in seasonal tropical agroecosystems under various management techniques. J. Appl. Ecol. 25: 595-607.

Masera O, Ordóñez M J, Dirzo R. 1992. Carbon emissions from deforestation in Mexico: current situation and long-term scenarios. En:Makundi W, Sathaye J, eds. Carbon emissions and sequestration in forests: case studies from seven developing countries. Energy and Environment Division, Lawrence Berkeley Laboratory publication LBL 32665. Vol. 4. Berkeley, California.

Mittermeier RA, Goettsch de M C. 1992. La importancia de la diversidad Biológica de México. En : Sarukhán J, Dirzo R, edrs. México ante los retos de la diversidad. Comisión Nacional para el Conocimiento y Uso de la Biodiversidad. Mexico, 63-73.

Raven PH, Evert RF, Curtis H. 1981. Biology of plants. 3rd. ed. Worth Pub. Inc. New York.

Rzedowski J. 1981. Un siglo de la botánica en México. Bol. Soc. Bot. México 40: 1-14.

Rzedowski J. 1991. Diversidad y orígenes de la flora fanerogámica de México. Acta Bot. Mex. 14: 3-21.

Rzedowski J, Calderón de Rzedowski G. 1987. El bosque tropical caducifolio de la región mexicana del Bajío. Trace 12: 12-21.

Sarukhán J. 1981. Mexico. En: Kormondy J, McCormick JF, edrs. Handbook of contemporary developments in world ecology. Greenwood Press, Westport, CT, 35-51.

Vovides A. 1981. Lista preliminar de plantas mexicanas raras o en peligro de extinción. Biótica 6: 219-228. 